East African Medical Journal Vol. 84 No. 3 March 2007

PROBLEMS IN DIAGNOSING ODONTOGENIC MYXOMA: CASE REPORT

F.M.A. Butt, BDS (UON), FDSRCS (Eng), Senior Registrar, M.L. Chindia, BDS (UON), MSc, FFDRCSI, Associate Professor, Oral and Maxillofacial Surgery and K.A. Wakoli, BDS (UON), MSc, FRSH (Eng), Lecturer, Oral Medicine and Pathology,

Faculty of Dental Sciences, University of Nairobi, P.O. Box 19676-00202, Nairobi, Kenya

Request for reprints to: Dr. F.M.A. Butt, P. O. Box 25361-00603, Nairobi, Kenya

\title{
PROBLEMS IN DIAGNOSING ODONTOGENIC MYXOMA: CASE REPORT
}

\author{
F.M.A. BUTT, M.L. CHINDIA and K.A. WAKOLI
}

\begin{abstract}
SUMMARY
Tumours and tumour-like growths arising from odontogenic tissues constitute a heterogenous group of lesions whose diagnosis can be particularly challenging on the part of both surgeons and pathologists. In children, these lesions are even more difficult to clinically and histopathologically diagnose definitively because of the concurrent diverse embryologic differentiation of dental tissues. A case is presented of a 12 year-old boy who was subjected to inappropriate primary management of a left mandibular mass, due apparently to inadequate consultation to establish an accurate diagnosis.
\end{abstract}

\section{INTRODUCTION}

Tumours and tumour-like growths arising from the odontogenic tissues constitute a heterogenous group of particularly interesting lesions, since they display the various inductive interactions that normally occur among the embryologic components of the developing tooth germ especially the mesenchyme (1). In children and adolescents, such neoplastic lesions are often benign and are of mesenchymal origin $(2,3)$. Choung and Kaban (4) were of the opinion that tumour histology in this age group did not correspond to their clinical behaviour. During this age of active development diverse lesions may present in the jaw bones arising from different tissues. These may include neoplastic lesions such as odontogenic myxoma, central giant cell granuloma; and tumour-like masses such as haemangiomas and fibrodysplastic conditions. Further, anaplastic lesions that may be malignant or mimic malignancy often occur in the growing jaw bones.

The origin of odontogenic myxoma (OM) is believed to be the mesenchyme of a developing tooth or periodontal ligament (5-7). Myxomas are predominantly found in young adults but may occur over a wide age range, the average age being 25 to
30 years. It rarely occurs before the age of ten years or after the age of 50 years. There is no particular gender predilection in the occurrence of the tumour (8). It is a slow growing non-capsulated and a locally aggressive tumour that causes bone destruction and soft tissue infiltration. It consists of an accumulation of mucoid ground substance with little collagen, the amount of which determines whether it can be called a myxofibroma or a fibromyxoma (9). Clinical presentation most commonly occurs in the second and third decades with the mandible affected more than the maxilla $(10,11)$. Radiologically OM may appear as a unilocular or multilocular radioluscency (soap-bubble pattern) with cortical expansion, tooth displacement and root resorption. Its clinical and radiologic behaviour poses potential difficulty when trying to arrive at a proper diagnosis.

A haemangioma histologically bears considerable resemblance to young granulation tissue and is nearly identical to some cases of pyogenic granuloma. They are both highly vascularised with endothelium lined channels engorged with red blood cells. However, a pyogenic granuloma is surrounded by a mixed inflammatory cell infiltrate of neutrophils, plasma cells and lymphocytes (5). 
On the other hand, anaplasia of tissues histologically, is characterised by undifferentiated or poorly differentiated cells. They have the presence of the primitive appearing unspecialised cells; and this may be the hallmark of malignancy. There is cell and neuclear pleomorphism, the neuclei are hyperchromatic and disproportionately larger than the cell; and there is loss of cell architecture with central areas of ischaemic necrosis. The aim of this article is to highlight the essence of systematic and appropriate investigations for the accurate diagnosis and management of paediatric jaw masses. Hence, a case is presented of 12-year-old boy who was subjected to inappropriate primary management of a mandibular mass due, apparently, to inadequate consultation to establish an accurate diagnosis.

\section{CASE REPORT}

A 12-year-old boy presented to the outpatient clinic at the University of Nairobi Dental Hospital (UNDH) on the $24^{\text {th }}$ November 2004, with a complaint of a swelling of the left mandible for a duration of four months; and a limitation of mouth opening. Apparently the swelling arose during the eruption of the first permanent mandibular molar. It was accompanied with dull pain and occasional bleeding as a result of occlusal trauma. With the exception of having been anaemic, his medical history was otherwise unremarkable. He was a fourth born in a family of eight healthy siblings without any history of a hereditary disease. His dental surgeon extracted the tooth en bloc with the associated swelling. Although healing of the extraction socket was uneventful the swelling reemerged and continued to enlarge progressively. A biopsy specimen revealed a histopathological picture of a haemangioma. Thereafter the patient was referred to an Ear, Nose and Throat (ENT) surgeon for further management. A repeat biopsy was done, and two specimens were taken to different Pathology laboratories where one reported it as a haemangioma and the second as a pyogenicum granuloma. Due to the inconsistency in the histopathology findings a fine needle aspirate was done and the specimen sent to a different laboratory where it was reported as an anaplastic tumour, upon which excision of the lesion was recommended. However, due to the inconsistent nature of the histopathology reporting of the lesion, further biopsy was done and reported as an ulcerated capillary haemangioma.

Within the period the patient was undergoing the multiple biopsy procedures, a computerised tomographic (CT) scan of the mandible was performed, which showed an expansile mass of the left ramus having broken the cortex with soft tissue extension. Two months later, a CT scan of the paranasal sinuses showed mucosal thickening of the left maxillary sinus. The report indicated a destructive bone lesion involving the left ramus of the mandible associated with a soft tissue mass and floating teeth. These features were reported as having been consistent with those of Burkitt's lymphoma. The patient was thereafter sent for radiotherapy for twenty days ostensibly based on the diagnosis of an anaplastic tumour. The swelling appeared to decrease in size but there was progressive limitation of mouth opening. The patient was advised that the lesion had become ready for resection with subsequent reconstruction. Hence, he was referred by the ENT surgeon to an oral and maxillofacial surgeon for further management.

On examination at the UNDH the patient had a diffuse swelling of the left mandible. The overlying skin was normal in colour, afebrile and was not fixed to the underlying tumour. The tumour margins were ill-defined and the mass was firm on palpation with no areas of tenderness. There was palpable submandibular lymphadenopathy bilaterally. Intra-orally mouth opening was limited at 15 to 20 $\mathrm{mm}$. Both the commissures had ulcerated lesions and the left buccal mucosa was pale and scarred. The swelling had expanded the ramus and the mandibular alveolus lingually involving the buccal cortex. The teeth on the affected side $(36,73,75)$ were mobile and tilted lingually. An orthopantomogram (Figure 1) showed a mixed early dentition with a multi-loculated radioluscency in the left ramus, coronoid process and angle of mandible. A clinical diagnosis was made of a benign intraosseous neoplasm, possibly an odontogenic myxoma or central giant cell granuloma.

As the lesion has been irradiated resulting in distortion of the tissue histology and the patient had been subjected to several incisional biopsies, a conservative excision of the lesion under general anaesthesia was planned, whence a partial mandibulectomy, sparing the condyle was accomplished. The postoperative recovery was 
unremarkable and the patient was discharged. A histopathological analysis of the surgical specimen showed a specimen of cellular fibrous connective tissue with significant vascular channels and myxomatous areas covered with abundant glycosaminoglycans. The surface was covered with hyperplastic keratinised stratified squamous epithelium. These appearances were consistent with those of an odontogenic myxoma (Figure 2).

\section{Figure 1}

An orthopantomogram showing a mixed early dentition with a multilloculated radioluscency in the left ramus, coronoid and angle of the ramus

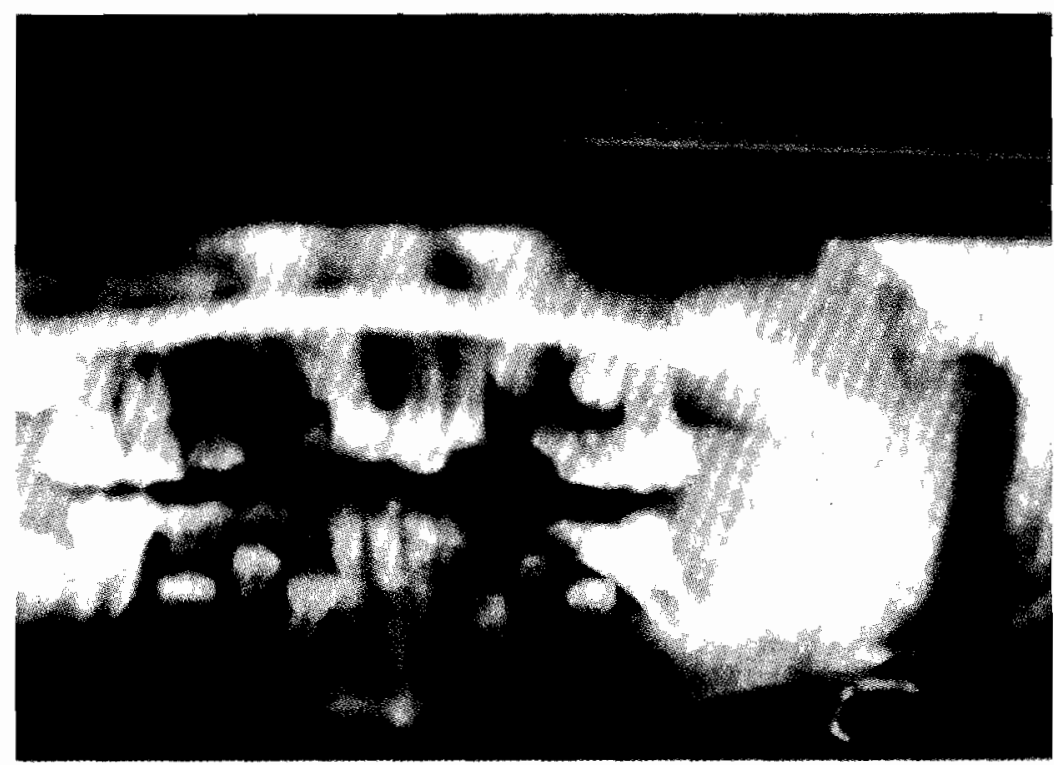

\section{Figure 2}

A photomicrograph showing a specimen of cellular fibrous connective tissue with significant vascular channels and myxomatous areas covered with abundant glycosaminoglycans. Haematoxylin and eosin stain.

(magnification $\times 100$ )

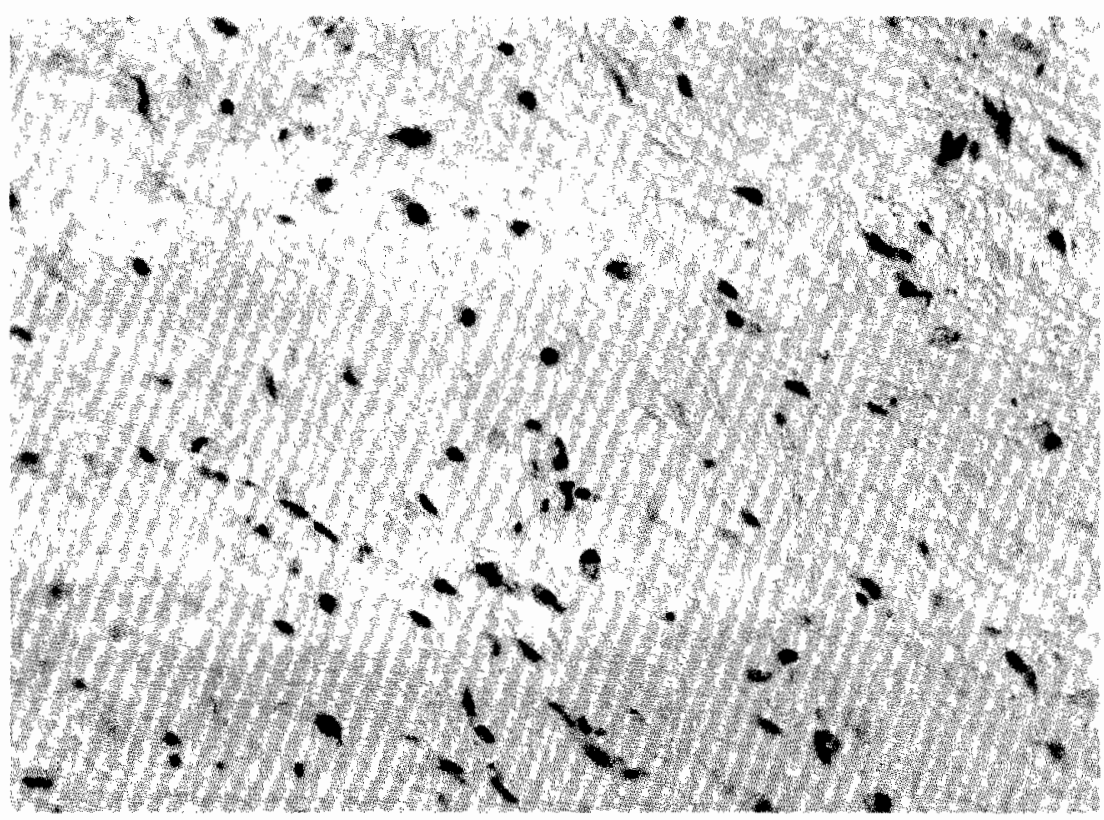




\section{DISCUSSION}

The jaws are unique structures with regard to the different pathological processes occurring within them. Multiple foci of embryonic tissues (ectodermal and neuroectodermal-mesodermal in origin) are contained within the jaws in utero and during the first 25 years of life. Nowhere in the skeletal system are undifferentiated embryonic structures present and active for so long a period of time after birth. Additionally, the jaws contain many scattered foci of odontogenic and non-odontogenic epithelial rests, with or without their associated mesenchymal anlage (12). These tissues possess the potential to develop into cysts or neoplasms in the postutero life, hence the vast diversity of maxillofacial lesions presenting especially in the younger age group. Therefore, odontogenic and non-odontogenic tumours in children present a diagnostic challenge due to their diverse mode of presentation in the absence of clinical symptoms apart from an obvious swelling. There is a dearth of data documenting these lesions particularly from the African continent thereby posing a dilemma for the practitioner who may not be exposed to this class of tumours. Hence, the incorporation of various specialties especially the Oral Pathologists and Oral and Maxillofacial surgeons who are specially trained to manage these tumours originating from odontogenic tissue is most likely to lead to accurate treatment. A comprehensive clinical history and examination with appropriate radiographs and biopsy from multiple sites would have been beneficial prior to any management of the present case.

Histological similarity between a haemangioma and a pyogenic granuloma is evident due to the resemblance with granulation tissue, however, reporting of an anaplastic tumour is at the extreme end of the spectrum and should have raised doubts in the surgeons mind in view of the clinical and radiological presentation of the lesion. It is possible to misreport a biopsy, especially if the size of the tissue was inadequate or a superficial part was incised with inflammatory cellular components and areas of necrosis. The site of biopsy is also crucial hence multiple sites should have been taken to understand the variation in the nature of the lesion. The pathologists may not have had adequate exposure to tissues of odontogenesis, hence an oral pathologist would have been handy to offer experienced interpretation of the pathology.
A misdiagnosis with incorrect treatment is detrimental to the overall patient management with grave consequences as was the case for this patient who had been subjected to radiation, a factor that could predispose to the development of malignancy. Prior to treatment it is the clinician's responsibility to employ a multidisciplinary approach to derive a definitive diagnosis for appropriate patient management. Therefore, a thorough understanding of odontogenesis and jaw development is essential in the study, correct diagnosis and management of jaw masses. The chronology of events clearly illustrate a discrepancy in the histopathological diagnosis and, therefore, a decision to subject the patient to irradiation was not justified at this point. In view of the bizarre scenario, consultation with the pathologists to consolidate the apparently erratic turn of events was imperative.

The primary management of the present case smacks of gross failure to observe the very basic professional tenet of adequate relevant investigation, interpretation and consultation in order to establish a definitive diagnosis for appropriate treatment. Sadly, it is quite apparent that this patient was only referred to a public university hospital for "further treatment" after the father pleaded that all his finances had been depleted in the payments for private professional fees and hospital care.

\section{ACKNOWLEDGEMENTS}

The authors would like to thank the Oral Pathologist Dr. E. Dimba, and laboratory technologists, Mr. J.O. Gichana and Ms. A.K. Limo for slide preparation and photography and the administration of the University of Nairobi Dental Hospital for permission to access the records for this case.

\section{REFERENCES}

1. Mosqueda-Taylor A., Ledesma-Montes C., Caballerosanoval S., et al. Odontogenic tumors in Mexico: A collaborative retrospective study of 349 cases. Oral Surg. 1997; 84: 672-675.

2. Asamoa E.O., Ayanlere A.O., Olaitan A.A. and Adekeye E.O. Paediatric tumours of the jaws in Northern Nigeria. I. Craniomaxillofac. Surg. 1990; 18: 130-135.

3. Ulmansky M., Lustmann J. and Balkin N. Tumours and tumor-like lesions of the oral cavity and related 
structures in Israel children. Int. J. Oral Maxillofacial Surg. 1999; 28: 291-294.

4. Choung E.R. and Kaban L.B. Diagnosis and treatment of jaw tumours in children. J. Oral Maxillofac. Surg. 1985; 63: 195-210.

5. Keszler A., Dominguez F.V. and Giannnuzio G. Myxoma in childhood. An analysis of 10 cases. Oral Maxillofac. Surg. 1995; 53: 518-521.

6. Mclough P.M. Odontogenic myxoma: An orthodontic presentation. Brit. Dent. J. 1991; 171: 212-213.

7. Van Der Waal I. Diseases of the jaws: Diagnosis and treatment. Copenhagen: Munksgaard. 1991; 206.

8. Neville B.D., Damn D.D., Allen C.M. and Bouquot J.E. Oral and Maxillofac. Pathol. $2^{\text {nd }}$ Ed. 2002; 635-637.
9. Farman A.G., Norte C.J., Grotespass F.W. and Van Zyi J.A. Myxofibroma of the jaws. Brit. J. Oral Surg. 1977; 15: 3-18

10. Slootweg P.J. and Wittkampf G. Myxoma of the jaws. An analysis of 15 cases. J. Maxillofac. Surg. 1986; 14: 46-52.

11. Peterson L.J., Indressono A.T. and Marciani R.D. Principles of oral and maxillofacial surgery. Vol 2. Philadelphia: J.B. Lippincott. 1992; 703-705.

12. Hoffman S., Jacoway J.R. and Krolls S.O. Intraossoeus and parosteal tumours of the jaws; $2^{\text {nd }}$ series 1987 ; 02-04. 